\title{
DATA PROVENANCE IN PHOTOGRAMMETRY THROUGH DOCUMENTATION PROTOCOLS
}

\author{
N. Carboni ${ }^{\text {a }}$, G. Bruseker ${ }^{\text {b }}$, A. Guillem ${ }^{\text {c }}$, D. Bellido Castañeda ${ }^{\text {d }}$, C. Coughenour ${ }^{\text {e }}$, M. Domajnko ${ }^{f}$, M. de Kramer ${ }^{g}$, M. M. Ramos \\ Calles ${ }^{\text {h }}$, E. K. Stathopoulou ${ }^{i}$, R. Suma ${ }^{j}$ \\ ${ }^{\text {a } U M R ~} 3495$ MAP CNRS/MCC, Marseille, France - nicola.carboni@map.cnrs.fr \\ ${ }^{\mathrm{b}}$ ICS-FORTH, Heraklion, Crete, Greece- bruseker@ics.forth.gr \\ ${ }^{\mathrm{c}}$ University of Ljubljana, Slovenia - Anais.Guillem@fgg.uni-lj.si \\ ${ }^{\mathrm{d}}$ ArcTron3D GmbH, Altenthann, Germany - dbellido@arctron.de \\ ${ }^{\mathrm{e}}$ Institute for Photogrammetry, University of Stuttgart, Germany - Chance.Coughenour@ifp.uni-stuttgart.de \\ ${ }^{\mathrm{f}}$ Fraunhofer Institute for Computer Graphics Research IGD, Darmstadt, Germany - matevz.domajnko@igd.fraunhofer.de \\ g7Reasons Medien GmbH, Vienna, Austria - mck@7reasons.net \\ h 3D Optical Metrology Group, FBK Trento, Italy - ramos@fbk.eu \\ ${ }^{i}$ Laboratory of Photogrammetry, National Technical University of Athens - elliestath@central.ntua.gr \\ ${ }^{j}$ University of Warwick, UK - r.suma@warwick.ac.uk
}

Commission V, WG V/2

KEY WORDS: Documentation, Metadata, Process Analysis, Close-range photogrammetry, Data Provenance, Paradata

\begin{abstract}
:
Documenting the relevant aspects in digitisation processes such as photogrammetry in order to provide a robust provenance for their products continues to present a challenge. The creation of a product that can be re-used scientifically requires a framework for consistent, standardised documentation of the entire digitisation pipeline. This article provides an analysis of the problems inherent to such goals and presents a series of protocols to document the various steps of a photogrammetric workflow. We propose this pipeline, with descriptors to track all phases of digital product creation in order to assure data provenance and enable the validation of the operations from an analytic and production perspective. The approach aims to support adopters of the workflow to define procedures with a long term perspective. The conceptual schema we present is founded on an analysis of information and actor exchanges in the digitisation process. The metadata were defined through the synthesis of previous proposals in this area and were tested on a case study. We performed the digitisation of a set of cultural heritage artefacts from an Iron Age burial in Ilmendorf, Germany. The objects were captured and processed using different techniques, including a comparison of different imaging tools and algorithms. This augmented the complexity of the process allowing us to test the flexibility of the schema for documenting complex scenarios. Although we have only presented a photogrammetry digitisation scenario, we claim that our schema is easily applicable to a multitude of 3D documentation processes.
\end{abstract}

\section{INTRODUCTION}

In contemporary cultural heritage, 3D digitisation, by means of photogrammetry, laser scanning and similar techniques, plays an important role in the documentation process. Digital documentation projects are required to provide products for end-users with different backgrounds, from engineers and computer science professionals to cultural heritage $(\mathrm{CH})$ researchers, managers and much more. The resulting 3D models, orthophotos or 2D plans can be used for various purposes, from geometric analysis of structures and features, to the creation of interactive visualisations in education, for providing support for management decision-making and information tracking. $\mathrm{CH}$ specialists increasingly rely on photogrammetric products for geometric documentation of an object and as a means to link and communicate knowledge across disciplines. Despite its increasing importance, recognised standards and protocols for the collection of the appropriate metadata and paradata that would support the effective longterm use of the photogrammetric output as a documentation resource are not broadly available. Image-based data and products should ideally stand as referenceable documents in their own right with a known provenance. This would allow another actor in the scientific research community to reuse them with reliable knowledge of the essential parameters that have gone into the creation and delivery of that resource. Appropriate documentation of such data objects' provenance would effectively put them on par with traditional bibliographic resources and thus open them up fully for academic and commercial re-use.

Several factors make this goal difficult to achieve. One of the most laborious tasks is organising and relating the different steps of a digitisation workflow. Proper organisation of the workflow has both important long and short-term effects on the data. The long-term effects concern the accessibility and reliability of the data that will be produced over time. Digitisation is considered to be an important mean of preserving artefacts by capturing important information from the original object. Yet, without preserving information about how the digitisation was generated, this aim is severely undermined, as the accuracy and reliability of the digitisation cannot be assessed. The workflow as a tool for day-to-day planning, meanwhile, focuses on the management of shared resources and their proper interpretation by the different members of a digitisation team. The continuous research into new applications of photogrammetric techniques using new technological tools means that novel ways of applying it to $\mathrm{CH}$ research are

* Corresponding author 
continuously being discovered, and this further complicates the goal of selecting the relevant metadata to record in the process. Finally, the task of defining protocols for digitisation workflow is made even more difficult by the multidisciplinary uses it is put to, requiring a dialogue among all the professionals involved to ensure that the protocol meets the minimum technical and analytic needs of each one to ensure its usefulness. Nevertheless, keeping a record of the digitisation process not only contributes to the sustainability of the produced data object, but also supports the long-term evaluation of techniques and methodologies, allowing the comparison of projected methods against results and the eventual refinement of techniques for various aims and relative to different types of objects.

Our proposed approach describes the essential processes to be carried out and the metadata to be gathered in digital photogrammetry survey projects, from image acquisition to the delivery of final products. The protocol was developed during the 3D digitisation process of an Early Iron Age burial discovered in 2010 during rescue excavations in Ilmendorf, Germany (Claßen et al., 2009; 2010). The finds encompass human remains and several large wood fragments as well as the grave goods that were the subject of this acquisition campaign. The latter consisted mainly of jewellery and beads made of gold, glass, amber, frit and bronze, averaging only a few centimetres in size. The artefacts are preserved and stored in the Archaeological State Collection in Munich, Germany. Our team was in charge of the $3 \mathrm{D}$ documentation of a selection of these objects, with the eventual goal of presenting the contextualised results in a digital interactive learning environment. The digitisation project's goals include both education and dissemination, as well as contributing to the preservation of the artefacts by allowing the public to interact with facsimiles of the objects without exposing the originals to any risk. As a complex multi-actor project, this case study is ideally suited to iteratively developing and testing this protocol.

In the following sections, we introduce our methodology, placing it in relation to previous works. Aspects of the case study are presented and discussed in order to illustrate how they were handled with regards to the workflow and protocols.

\section{METHODOLOGY AND BACKGROUND}

Reality-based 3D modelling is a well-established and low-cost process that can typically be divided into the following steps: project planning (equipment, staff, budget, time, etc), data acquisition (image and reference system observations) and data processing (from images to point clouds and photorealistic 3D models) (Remondino et al., 2013; Remondino and El-Hakim, 2006). The photogrammetric process - especially when dealing with cultural heritage objects - requires the coordinated efforts of different actors as well as the integration of different kinds of data. We propose a typical workflow for a complete digitisation, breaking it up into seven iterative and repeatable steps: (i) project planning, (ii) preliminary studies, (iii) data acquisition, (iv) data processing, (v) result analyses and acceptance testing (vi) creation of dissemination products and (vii) delivery. For each step, we propose an information workflow methodology and metadata schema for capturing the data relevant to each step. The complete collection of registers, as well as a sample of control lists developed during the project, is available online at http://itndch.map.archi.fr/pacs.

Each of the individual steps of the overall workflow is the result of an extensive process of analysis of the implicit and explicit information relied upon and the general action/decision events that occur during the production and exchange of data in a digitisation process using photogrammetry. To represent the workflow, we adopted the Business Process Modeling Notation 2.0 (BPMN) (White, 2004; Chinosi and Trombetta, 2012) and encoded the general picture into seven Business Process Diagrams (BPD). BPD were chosen because they are a well known tool to create a formal analysis of relevant events, allowing us to show how the different participants interact and to indicate at what point data and metadata should be captured to document essential information with regards to the creation of digital products. It is especially useful in this latter process of helping visualise and define when, within the workflow, documentation should be created and updated, as well as who is in charge of doing so, providing a well-defined information pipeline within the digitisation team that can be used as a guide for the organisation of the overall process (Figure 1,2 and 4).

Each of the BPD we propose is accompanied by a set of metadata registers that indicate a schema of fields to be used for the description of the relevant information called for in each step. These registers together form a conceptual schema which can easily be encoded in various languages such as XML (eXtensible Markup Language) or DDL (Data Definition Language) according to individual projects' needs. An example of encoding this data into a database schema is also provided in the additional documentation available on the website noted above.

\subsection{State of the art}

A number of previous projects have taken into account the documentation of the photogrammetric workflow, either focusing on the construction of basic metadata for describing the overall activity, or concentrating on the acquisition and processing steps.

TAPEnADe (Tools and Acquisition Protocols for Enhancing the artefact Documentation), a joint project between IGN, CNRSMAP and FBK (Nony et al., 2012; Pierrot-Deseilligny et al., 2011) falls into the latter category. It aims to provide methodologies and best practices for a typical digitisation pipeline applicable to different cultural heritage contexts. It includes an overview of the entire process, from the shooting positions to $3 \mathrm{D}$ point cloud generation. The proposal of a table to record the shooting information is most relevant to our work. CARARE (Fernie et al., 2013) was a European project that focused on the aggregation of content from the archaeological and architectural domain. The project initially proposed a metadata schema, further developed during the 3D ICONS project, that would take into account the different media and representations for a cultural object as well as the activities that produced them. CARARE is based on various standards, like MIDAS, LIDO, POLIS DTD and CIDOC-CRM and it is aligned with EDM (Europeana Data Model) (D'Andrea and Fernie, 2013). The main focus is on the cultural object and its representation - for example, strong attention is given to the geometry of the digital model. An activity documentation unit is proposed that can be used to describe the photogrammetric process. Unfortunately, the details of this process and the specific parameters used are not specified. Only two fields, "Methods" and "Technique", are available for this task, and they cannot fully capture the complexity of the process. Moreover, the processing techniques inherent to photogrammetric work are not part of the schema, leaving out important information for tracing the overall provenance and evaluating the scientific value of the digital output.

Another example, more focused on the construction of 3D data, is the STARC Metadata Schema, developed by Ronzino et al. 
(2012). The presented documentation units allow for a detailed description of the project, acquisition activity, data processing and publication of the final product. It is well constructed, and goes a long way in defining detailed metadata and paradata for a digitisation process, but its scope covers only the data provenance for a 3D object repository and doesn't include the possibility of linking the research activity with the technique used to resolve a particular research question.

The above-mentioned works provide a basic account of the provenance and interpretation of the data, but do not supply their users with the necessary tool for exploiting the information silos frequently hidden in their repositories. To fulfill this objective, we must analyse the diverse units and the type of relationships between the different steps of the digitisation process, their primitive interconnections and the functional requirements of the data elements recorded.

\section{A PROPOSAL FOR A PHOTOGRAMMETRIC DOCUMENTATION WORKFLOW PROTOCOL}

The steps of our documentation protocol (Figure 1) are described in the following sections. Their development and evaluation, both in the office and then in the field with a real case study, allowed us to immediately test and progressively adjust the proposed model, deciding on the key information to be recorded and preserved. At the same time, we were able to discover which data were not important, allowing us to streamline the metadata recording process as much as possible. The final proposal is the fruit of interdisciplinary research between 3D surveying experts, technicians, $\mathrm{CH}$ analysts and dissemination professionals.

We are only able to present here a selection of the workflow diagrams and metadata forms that we created. For each step of the protocol, we will present the rationale of the step, and give an example of how following the workflow and recording the relevant metadata aided in the management of data production and exchange amongst the digitisation team in our case study. For some of the steps, we have been able to include the relevant BPDs or extracts from completed metadata registers. Please visit the site referenced above for access to the complete documentation set.

\subsection{Project Planning}

photogrammetric workflow, and digitisation more generally, is the project itself, to which all intersecting and related activities, physical objects, digital documents, actors and equipment can be referenced. Further, each digitisation project should be defined in relation to a goal, or is initiated in order to answer one or more research questions. This goal-oriented nature of the digitisation process is a natural part of any scientific activity, but is often documented only in narrative form or, in the worst cases, remains implicit in the minds of the actors initiating the project. Therefore, in addition to recommending the recording of the originating project of the digital object, we make the proposal to record also the questions/goals that spurred the project. These questions are to be referenced later in the workflow in order to link questions to types of solutions as well as their success or failure at resolving the questions posed. In this way, the digitisation process contributes to its own progressive amelioration through long term recording of successful and unsuccessful strategies relative to different types of goals.

In the Ilmendorf case study, the Project Register was useful for recording the actors involved (engineers, cultural heritage specialists and managers) and the artefacts under consideration. The equipment register allowed for the recording of available equipment which, in our case, included full-frame DSLR cameras with multiple focal length lenses, studio lights, tripods, a structured light scanner, inter alia.

Explicitly recording the research question of the case study proved to be one of the biggest challenges. Explicit recording of this information in a formalised manner was not a part of the usual digitisation practice, with the questions being implicitly understood. In fact, rendering the research questions explicit and available to all members of the team in the Question Register generated useful and interesting dialogue. Questions and research aims included the testing of three different photogrammetry software applications (Photoscan, SURE, and aspect3D) and exploring the use of High Dynamic Range (HDR) imaging in photogrammetry as compared to standard images. We also planned to reuse the digital models for dissemination. For each of these aims, we also documented the projected acquisition, processing, and dissemination methods which would be required in order to organise the overall workflow.

\subsection{Preliminary Studies}

Any kind of digitisation project, using photogrammetry or otherwise, must take into account previous and parallel work regarding not only

\begin{tabular}{|c|c|c|c|c|c|c|}
\hline $\begin{array}{c}\text { Step I: } \\
\text { Project } \\
\text { Definition }\end{array}$ & $\begin{array}{c}\text { Step 2: } \\
\text { Preliminary } \\
\text { Studies }\end{array}$ & $\begin{array}{c}\text { Step 3: } \\
\text { Data } \\
\text { Acquisition }\end{array}$ & $\begin{array}{c}\text { Step 4: } \\
\text { Data } \\
\text { Processing }\end{array}$ & $\begin{array}{l}\text { Step 5: } \\
\text { Analysis }\end{array}$ & $\rightarrow \quad \begin{array}{c}\text { Step 6: } \\
\text { Dissemination }\end{array}$ & $\begin{array}{l}\text { Step 7: } \\
\text { Delivery }\end{array}$ \\
\hline$\uparrow$ & $\mid \uparrow$ & $\mid \uparrow$ & $\mid \uparrow$ & $\uparrow$ & $\uparrow$ & \\
\hline $\begin{array}{l}\text { Central Register: } \\
\text {-Project } \\
\text {-Question }\end{array}$ & $\begin{array}{l}\text { Central Register: } \\
- \text {-Research } \\
\text {-Sitie Visit } \\
\text {-Bibliographic }\end{array}$ & $\begin{array}{l}\text { Central Register: } \\
\text {-Acquisition Project } \\
\text {-Acquisition Activity } \\
\text {-Equipment Setup } \\
\text { Parameters } \\
\text {-Object Acquisition } \\
\text { Configuration }\end{array}$ & $\begin{array}{l}\text { Central Register: } \\
\text {-Processing Project } \\
\text {-Processing Plan } \\
\text {-Data Processing }\end{array}$ & $\begin{array}{l}\text { Central Register: } \\
\text {-Product Evaluation } \\
\text {-Answer Evaluation } \\
\text { Register }\end{array}$ & \begin{tabular}{|l|} 
Central Register: \\
-Dissemination \\
Project \\
-Product \\
Development Plan \\
-Dissemination \\
Product Development \\
Activity
\end{tabular} & $\begin{array}{l}\text { Central Register: } \\
\text {-Client Individual } \\
\text { Product Feedback, } \\
\text {-Dissemination } \\
\text { Feedback }\end{array}$ \\
\hline $\begin{array}{l}\text { Supporting Register: } \\
\text {-Objects } \\
\text {-Actors } \\
\text {-Digital Assets }\end{array}$ & $\begin{array}{l}\text { Supporting Register: } \\
\text {-Question } \\
\text {-Actors } \\
\text {-Digital Assets } \\
\text {-Project }\end{array}$ & $\begin{array}{l}\text { Supporting Register: } \\
\text {-Equipment } \\
\text {-Actors } \\
\text {-Project } \\
\text {-Digital Asset }\end{array}$ & $\begin{array}{l}\text { Supporting Register: } \\
\text {-Question } \\
\text {-Actors } \\
\text {-Object } \\
\text {-Digitial Assets } \\
\text {-Equipment }\end{array}$ & $\begin{array}{l}\text { Supporting Register: } \\
\text {-Question } \\
\text {-Actors } \\
\text {-Object } \\
\text {-Digital Assets } \\
\text {-Bibliographic }\end{array}$ & $\begin{array}{l}\text { Supporting Register: } \\
\text {-Object } \\
\text {-Actors } \\
\text {-Digital Assets } \\
\text {-Bibliographic } \\
\text {-Equipment } \\
\text {-Site Visit }\end{array}$ & $\begin{array}{l}\text { Supporting Register: } \\
\text {-Question } \\
\text {-Dissemination } \\
\text { Project } \\
\text {-Bibliographic }\end{array}$ \\
\hline
\end{tabular}

Figure 1. Overview of the general documentation pipeline and its related registers the geometric documentation of the object but the relevant literature and information from

contemporary site visits as well. The data collected in the preliminary study step serves to inform all the following processes, both as a reference and as potential input to the planning and processing stages. 
We therefore proposed the preparation of a Research Register to explicitly document this background research and to stand as a base resource in the project.

This Research Register can be linked to Bibliographic Register, previous geometric documentation in the Digital Assets Register and the Site Visit Register.

In our case study, the site and objects had not previously been geometrically recorded but several related articles were collected in the Bibliographic Register. Instead of a site visit, given that the site was a rescue excavation, local experts explained the context of the artefacts. The presentation document was appended to the Site Visit Register. Taking this additional research step and documenting it explicitly was of use both to the acquisition team in planning its acquisition strategies and to the dissemination team in preparing the application for presenting the finished models in their full context.

This step is represented in the figure below.

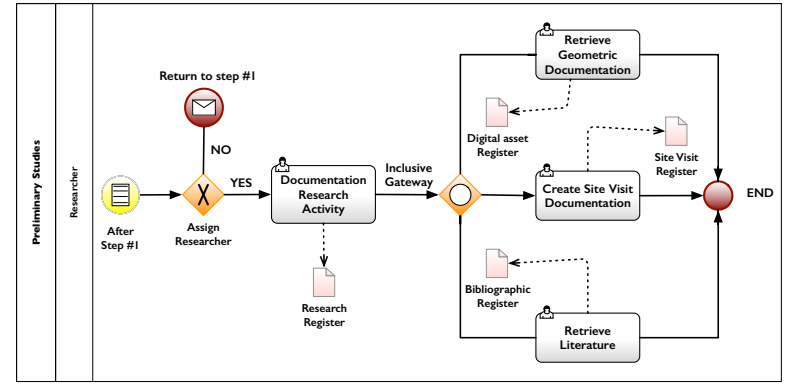

Figure 2. BPD: Step 2

\subsection{Acquisition}

The acquisition phase attempts to follow the plan established during the initial setup of the project, taking into account the projected output requirements to answer the research questions and goals posed. This phase requires careful and detailed documentation in order to fully contextualise the data acquired, and overall, to make them reusable for the different current and future actors engaged with the data. The central documentation tool for this phase is the Acquisition Project Register, understood as a container activity for documenting multiple acquisition activities. The aim of each object's acquisition should therefore be registered separately, documenting the object of analysis, the level of accuracy required, the agents involved and the relevant time limits.

The individual acquisition activities should be documented separately in the Acquisition Activity Register, using an activity-based metadata log linked to the overall acquisition project. Each data object produced during an acquisition event is the result of the activity of some actor(s) in a certain environment at a certain time, and involves the use of specific equipment, set to particular parameters. Recording these parameters correctly is of paramount importance to allow its interpretation and derive the desired results. The acquisition activity itself should be recorded with regards to: its object (e.g. the artefact or the site under study), acquisition time and environmental factors, actors involved, equipment employed (linked to its technical details) and setup parameters during the capture. These parameters involve the shooting methodology, overlap, calibration and other decision factors which affect the overall outcome of the final digital product (GSD, DoF, distance to the object etc.). As an example, the acquisition parameters for an individual object (more detail below) of our case study are recorded in Tables 1 and 2.
Finally, each acquisition activity is linked to its products, which are recorded in the comprehensive Digital Asset Register.

Documenting the acquisition and its relevant parameters proved one of the most challenging processes to discretise into its basic components.

\begin{tabular}{|l|l|}
\hline \multicolumn{2}{|c|}{ Equipment Setup Parameters Register - Camera } \\
\hline Camera Body & Nikon D800 \\
\hline Lens & Nikkor 28 \\
\hline Lens Type & wide angle \\
\hline Platform & tripod \\
\hline Orientation & N/A \\
\hline ISO & 100 \\
\hline Shutter mode & manual, self-timer \\
\hline Shutter speed (sec) & $1 / 3$ \\
\hline Aperture (f-stops) & 14 \\
\hline Focus & manual \\
\hline Focus fixed & YES \\
\hline Focus distance & $33 \mathrm{~cm}$ \\
\hline Depth of field & $12 \mathrm{~cm}$ \\
\hline Near focus & $28 \mathrm{~cm}$ \\
\hline Far focus & $40 \mathrm{~cm}$ \\
\hline Focal Length (mm) & $28 \mathrm{~mm}$ \\
\hline White balance & YES \\
\hline White balance pre-set & grey card \\
\hline White balance methodology used & custom WB \\
\hline Lightmeter used & NO \\
\hline $\begin{array}{l}\text { Camera Pre-Calibrated } \text { (geometric } \\
\text { calibration) }\end{array}$ & NO \\
\hline Color calibration & YES \\
\hline HDR & YES \\
\hline HDR technique & bracketing \\
\hline HDR Bracketed series & 3 \\
\hline HDR exposure values & $-1 \mathrm{EV}, 0,+1 \mathrm{EV}$ \\
\hline Tabl 1 Form filled for Equip & Par Segist - \\
\hline
\end{tabular}

Table 1. Form filled for Equipment Setup Parameters Register Camera

\begin{tabular}{|l|l|}
\hline \multicolumn{2}{|c|}{ Object Acquisition Configuration Register } \\
\hline Camera Configuration Type & convergent \\
\hline $\begin{array}{l}\text { Average GSD (Ground Sampling } \\
\text { Distance) }\end{array}$ & $0.06 \mathrm{~mm}$ \\
\hline $\begin{array}{l}\text { Percentage of Object Covered } \\
(\%)\end{array}$ & 80 \\
\hline $\begin{array}{l}\text { Average Distance From The } \\
\text { Object }\end{array}$ & $33 \mathrm{~cm}$ \\
\hline Forward/longitudinal Overlap (\%) & 80 \\
\hline Side Overlap (\%) & - \\
\hline Markers & YES \\
\hline Markers type/codification & calibration pattern codified \\
\hline Markers no. & 96 \\
\hline GCP & NO \\
\hline On-site Camera Calibration & NO \\
\hline Image base/baseline & $16 \mathrm{~cm}$ \\
\hline Obstacles & NO \\
\hline
\end{tabular}

Table 2. Form filled for Object Acquisition Configuration Register

In the table above we described the custom setup which was used to optimise the photogrammetric data acquisition, which in our case included a full-frame DSLR camera, Nikon D800, with a fixed $28 \mathrm{~mm}$ focal lens, which was mounted on a tripod with a self-timer employed for shooting with stability. The camera resolution was set to the maximum $(7360 x 4912)$, while manual exposure and manual focus (spot) were used to fully control the acquisition process. Both the camera and the tripod were 
previously entered in the general Equipment Register, so as not to repeat the information in the acquisition step and to better relate the equipment with the products of the acquisition and the actors involved.

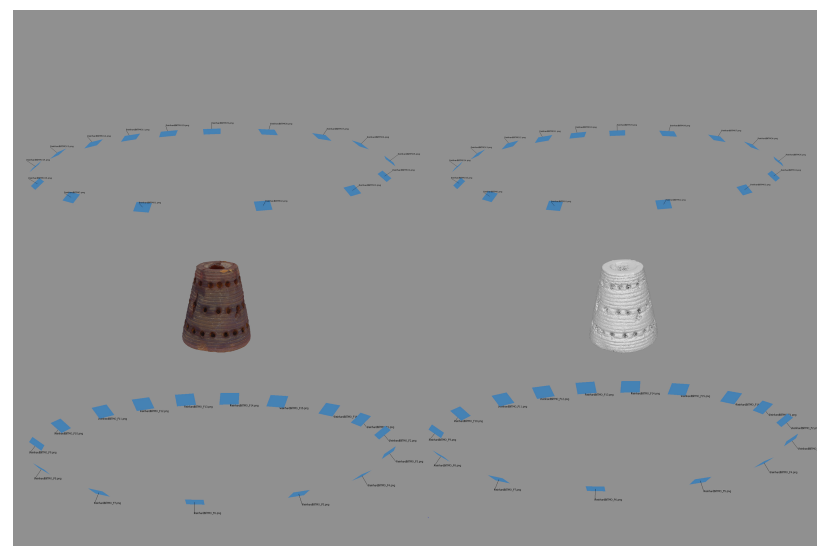

Figure 3. Camera positions related to the object.

In this case, the objects to be acquired were placed on a turntable, resulting in convergent acquisition (Figure 3). Within the model, we take into account the input of $n$ images for the photogrammetric reconstruction in order to link the original file to the final outcome of the reconstruction. We do not explicitly document the spatial position of the camera in relation to the object plane because such parameters are later automatically calculated by the software. The turntable allowed us to keep the camera parameters fixed for each object. The use of multiple set of parameters should be documented separately in the Equipment Setup Parameters, but as this is a reference model it is for users to decide the exact level of granularity they want to pursue in recording these details.

Recording the time taken allows us to evaluate the speed of the digitisation in comparison to other setups and rate their efficiency. While in our case, we tested the protocol only in relation to small scale objects, there is no issue for the scalability of the approach to large scale object. We use the activities of processing as the units of documentation that together give the provenance for a digitised object. Therefore, it is irrelevant to our methodology whether the object to be digitised is large or small because in the former case it can be still documented into its smaller processing units, which are in the processing phase registered together.

In addition to the setup information shown in Table 1 and 2 , the protocol includes descriptors for specifying the environmental light control, as well as the presence of markers during the acquisition event. The registration of the different exposure values (bracketing) that will later be used for post-processing HDR image generation is also crucial. Through the use of our documentation registers, we were able to record the individual photogrammetric data acquisition activities and their input parameters, notably equipment and acquisition methodology, and link these to the digital outputs. This allows us not only to contextualise the data acquired but also to provide more general information on field-tested setups for future scenarios with similar conditions.

It is important to underline that the recording of certain types of parameters does not have to be carried out manually, but can easily be automated. While this interest in automated metadata collection does not concern only the acquisition phase, we noticed these kinds of requirements and requests come out specifically in relation to this specific phase.

\subsection{Processing}

The processing step takes the results of data acquisition activities and plans for their transformation using automatic or semi-automatic software operations. The results are data products aiming to meet the specifications set out during the initial evaluation of the research question as established in the project planning step. Since processing can be a complex operation, requiring continuous iterative 'results versus goals' validation, we propose a three-tier documentation scheme to track these tasks: (i) Processing Project Register, (ii) Processing Plan Register, and (iii) Data Processing Register.

The overall documentation unit is the Processing Project Register, which acts as a wrapper for a sequence of processing actions that will take place according to a documented plan held in the Processing Plan Register. Moreover, the Processing Project Register, outlines the products and its accuracy expected, and by whom the processing will be organised, controlled and performed. The processing action links to the original question to which the processing plan should respond, as illustrated in Figure 4.

The actual execution of a processing project that will result in the intended end product will require several processing steps with individual processing plans and iterative processing actions checking for data accuracy and acceptability.

For each discrete step, we envision a separate documentation of a data processing plan, which would lay out the software, hardware and methodology to be employed to achieve the desired end. Each data processing action that is carried out under a plan is then documented in its own right in the Data Processing Register with the relevant input, output and

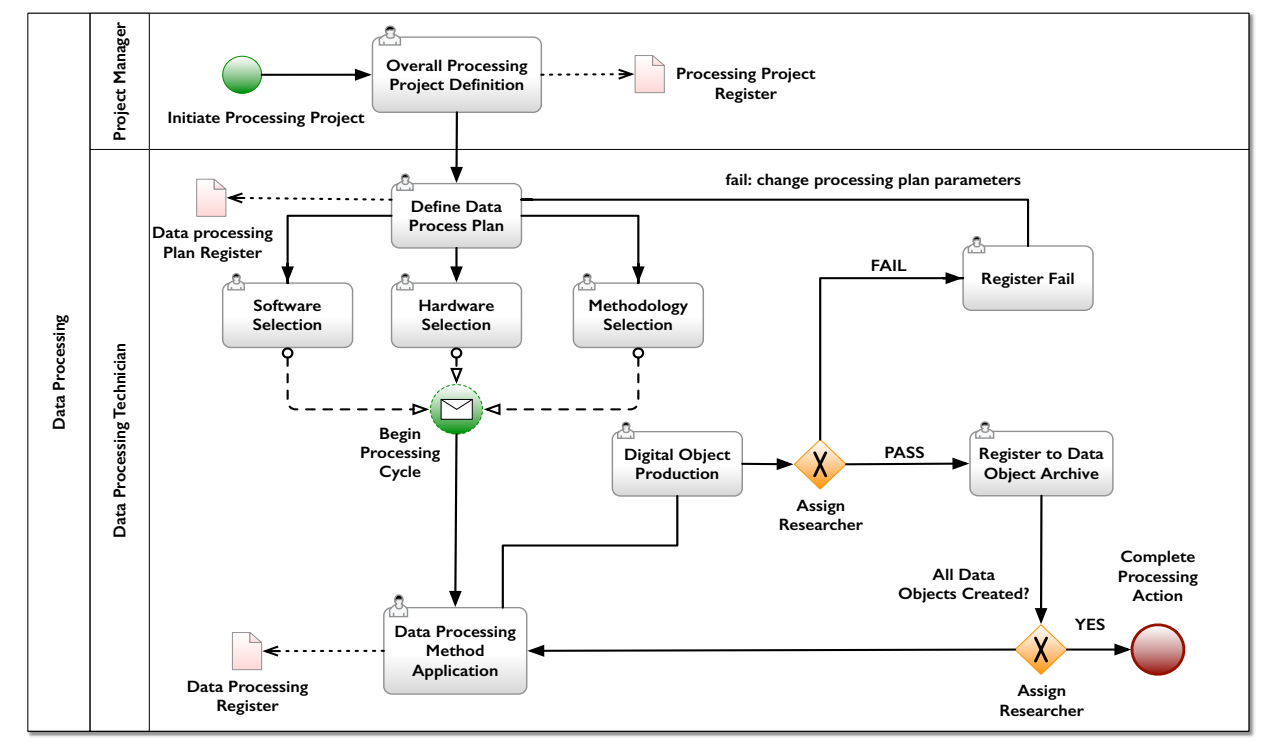

Figure 4. BDP: Step 4. 
indicated parameters used and obtained.

As an example, a type of data processing project is the overall creation of HDR tonemapped images from bracketing, while the processing action is the discrete operation taken for achieving a goal such as the HDR image generation and the subsequent tone mapping operation. These actions used different parameters that need to be recorded in order to register the provenance of the data as well as comply with the need for reproducibility of results in the future.

Furthermore, the differentiation of processing plan and processing action is useful to establish the similarity or dissimilarity between the original plan and the real results of the processing. The execution of the processing action can result in different outcomes than the original expectations and therefore need to be adjusted. The recording of different approaches allows the documentation of not only success but also errors, useful as a foundation of the knowledge organisation of a research group. The documentation of the obtained accuracy is essential, as it determines the success or failure of the process. Success validates the data processing plan and allows the articulation of the next step of the processing project. Failure indicates that the data processing actor/engineer should return to the processing plan, adjust relevant parameters, and then run the processing actions again until a satisfactory result can be obtained, or a negation of one of the relevant factors can be obtained (e.g. software, hardware, methodology or input variables)

In this case study, we recorded the processing of exposure bracketing data with the HDR Toolbox in Matlab (Banterle et al., 2011). The three different exposures were first used to generate a single HDR image and then the image was tonemapped using (Reinhard et al., 2002) (Figure 5). The possibility of documenting the algorithms and parameters adopted during the processing step and evaluating the success/failure of the latter sequence of actions was seen as a key facility provided by the proposed protocol and registers. It enabled us to build an accurate analysis of the processing cycle, which was recorded and linked to the current processing configuration in order to build up a data-driven library of parameters and acquisition types.

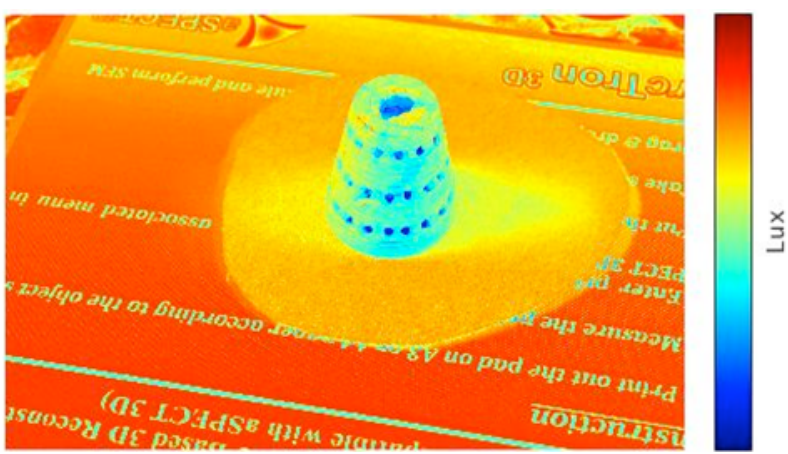

Figure 5. False color image of the Amber Object luminance values for HDR image

The three-step approach to documenting data processing allowed us to capture the array of different algorithms and software platforms that were tested to obtain optimal results. The images were initially aligned in Agisoft PhotoScan and aspect3D. Dense image matching, mesh generation and texture mapping were performed in the former as well as in SURE (Rothermel et al., 2012) software. Three different processing projects were created in order to discretise the complexity of the operations and achieve a better understanding and record of the distinct operations performed and the resulting data
As mentioned in section 3.3, images were acquired for both sides of each object. All 32 of them (16 for each side) and their masks were used in the bundle adjustment step and successfully registered and aligned (Figure 3 and 6). After dense image matching, a final point cloud was generated (Figure 7, with 500,000 points). Textured $3 \mathrm{D}$ meshes of the object were also produced (Figure 8). Each of these steps was registered as a different processing plan and activity in order to comply with the provenance principles of the documentation framework. In order to establish a better accountability for the steps taken and influencing factors, the algorithms used were also recorded whenever possible.
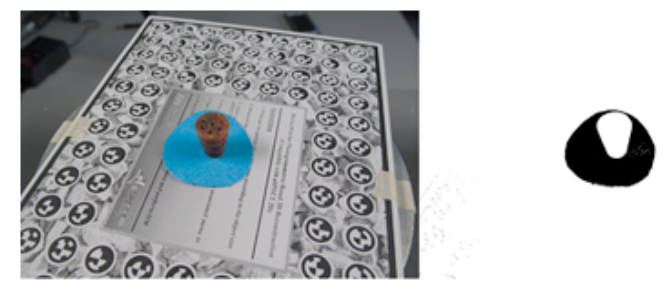

Figure 6. Masking steps of object images for successful alignment from different sides

Testing of the metadata forms revealed that we could accurately and analytically capture all data processing steps. While time consuming to fill, the result of recording the metadata and paradata for this step was a much easier and more transparent discussion of the results between the experts involved. Furthermore, it helped to identify some processing errors.
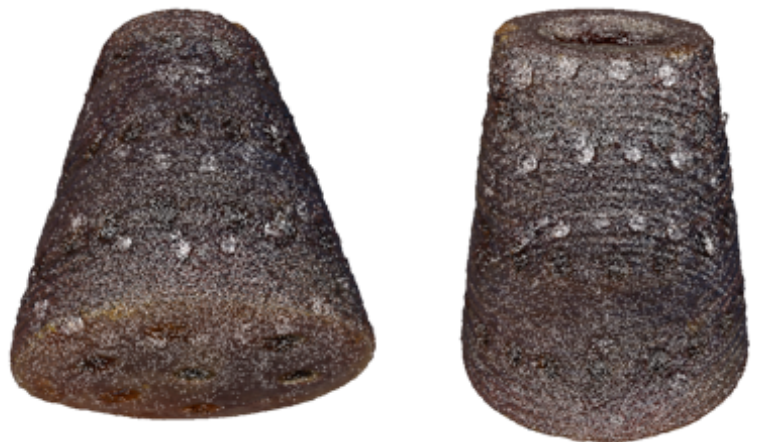

Figure 7. Point cloud of one of the processed objects based on 32 images, two datasets from the rotated object.

\subsection{Analysis and Acceptance Testing}

After the completion of a cycle of acquisition and processing, we introduce in the protocol an analytical phase in which the resultant products are tested for their potential usefulness in regards to the initial research questions posed. Here, we envision specialists in a team or even clients, engaging in a review process of the raw acquisition products and/or processed data. We break up the evaluation and analysis stage into two principal components: (i) a general Product Evaluation Register documented on a per question basis, and (ii) a specific Answer Evaluation Register for each question in which the individual data objects generated to answer that question are evaluated.

For each stated research question, an analysis form can be filled out giving an overview of the digital objects produced and an overall rating of the methodology adopted and the usefulness of the data generated. Here, we have in mind a comparison of the objects against broader and less technical criteria than those envisioned in the self-testing aspects of the acquisition and processing steps of the digitisation project. The question is to 
understand whether or not the outputs were able to support an interpretation relevant to the original research questions. Regardless of the result, a documentation of the evaluation of the methods employed and the method of evaluation helps to improve the process of answering future questions.

In the second, more specific step, the individual data products related to the question are accepted or rejected, with a documented justification. This step, therefore, can potentially result in restarting the processing project or even the acquisition, depending on the outcome.

This review phase was conceived to create a formal space for documenting the analysis and testing of the processed models as digital assets and link their evaluation to the initial research questions.

A particularly interesting case for discussion and analysis was the additional use of tone-mapped HDR images as an input to the photogrammetric software for the generation of our $3 \mathrm{D}$ models. In our particular case study, there was not any significant improvement on the sparse point cloud, whereas the visual appearance of the final model itself was slightly improved.
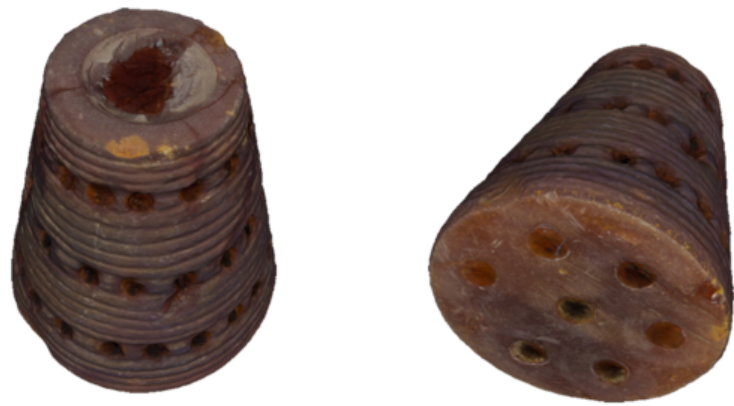

Figure 8. Final 3D model accepted for one of the processed objects.

Although conclusive data evaluation for this technique cannot yet be presented, documenting our question and preliminary results helps to inform a longer term scientific process, ensuring the availability of information for further statistical studies and repeatability of the experiments. This offers future researchers a basis upon which they could build to draw their own conclusions and develop their own research.

\subsection{Dissemination}

Dissemination plays an ever more prominent role in cultural heritage research and in some cases it may even be the raison d'etre for the entire project. A careful balance is therefore required; while a project must ensure from its inception that it will produce results that can be used for the desired dissemination, data reusability means that information that may not directly affect the dissemination must also be recorded to ensure its reliability and usefulness in the future. Which products are requested and required for the dissemination aspect of digitisation can vary widely, depending on the intended audience, budget, space constraints and other factors. From a model for simple visualisations for the general public to fullscale, 4D interactive environments, the dissemination of the results of a digital documentation project could be the subject of an entire workflow control process of its own. However, we propose to treat dissemination activities as an integral part of the total digitisation workflow. Considered from this perspective, the dissemination control process can be modelled using a framework similar to the processing structure, differing mostly in its consideration of goal variables. While processing must consider its base questions of accuracy, dissemination must consider questions of suitability for an audience.

Therefore, we propose a parallel tripartite structure to the processing control activity. This consists of the basic documentation units of the Dissemination Project Register, Product Development Plan Register and Dissemination Product Development Activity Register.

The overall Dissemination Project Register records the target audience, the planned medium, the aim of the dissemination product and the projected dates of production. Each dissemination project will entail a number of internal development cycles that will require their own planning. In the dissemination cycle, the developers can take full advantage of all generated products within the digitisation pipeline to this point. They may therefore import assets from the research, acquisition and processing phases as part of the base materials from which to construct their final product. This provides a map of provenance of the inputs to the end product, while not imposing overly onerous metadata entry requirements on the user. Each individual product of the dissemination cycle should be subject to at least one planning event. These serve as the control base from which to execute processing for the development of dissemination products. As in the acquisition phase, we suggest that each significant processing event for the creation of a digital asset related to dissemination purposes should be documented and related to its relevant Product Development Plan and the activities initiated to achieve that plan recorded in a Dissemination Product Development Activity Register.

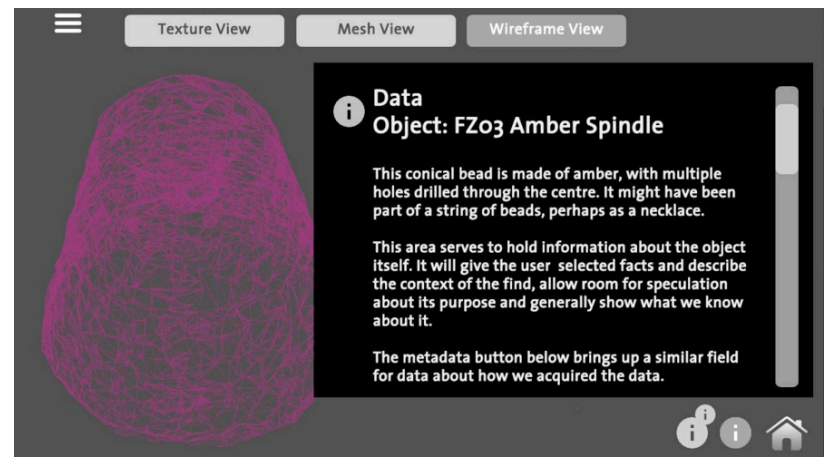

Figure 9. Screenshot for a dissemination app showing a detailed object view - artefacts can be displayed in 3D as textured, meshed, or wireframe views; buttons open text fields with data and metadata for each

In our case study, the digital dissemination outcome suggested for Ilmendorf was a web-based application running in the Unity3D game engine (Figure 9). By providing virtual interaction, it enables the user to investigate individual objects in greater detail than seeing the physical object in a restricted environment would allow. The scientific accuracy of this output is strongly supported by the workflow protocol we adopted, as all elements presented through this game-like experience, can be traced back through the processes that were used to generate them, all the way to the original artefact.

\subsection{Project Conclusion and Delivery}

The completion of a digital documentation project is signalled by the handing over of the requested final products to the client, be it an internal researcher or external contracting actor, who should provide an evaluation, whose acceptance or rejection of the end products should be recorded, and may trigger the 
development of a new process for acquisition, processing and dissemination product creation. The evaluation of the dissemination products should be recorded in the Client Individual Product Feedback and Dissemination Feedback Register and should follow the same model as that of the analysis and evaluation step for acquisition and processing steps, only now with regards to final dissemination products and the overall project goals. The recording of such information is to be considered vital, because it will enhance the quality control with regards to the digitisation steps needed.

\section{CONCLUSION}

Our proposal allows for the tracking of the digitisation process that leads to and makes possible the digital product through the complete photogrammetric workflow from planning through acquisition, processing and analysis all the way to dissemination. We outline our complete workflow and relate some of the key metadata registers we propose to capture the main framework of data interactions, from the functional requirements to the scientific analysis of a digital documentation project. The model we propose has been elaborated chiefly in relation to photogrammetric acquisition but has been designed to be general and flexible enough to be used for different acquisition methods (e.g. laser scanning), as well as easily integrated with other schema for the object description (MIDAS, CDWA) or digital preservation (PREMIS). The account presented above leaves open future research into encoding of the metadata into particular schemata and furthermore into an ontological framework. The modelling of the latter requires more comprehensive testing on sample data from a dedicated community and can be achieved only through a constant collaboration between different institutional actors. Our aim here is to start the conversation from its foundation: the interactions that create the data. For this reason in each step, we have shown how the documentation contributed to the overall understanding of the product both as a practical tool for sharing information among interdisciplinary team members during the execution of the project and as a contribution to the long-term understanding of the products created.

\section{ACKNOWLEDGEMENTS}

This project has received funding from the European Union's Seventh Framework Programme for research, technological development and demonstration under grant agreement no 608013.

We would like to thank the company ArcTron3D GmbH which provided the different equipment used in the case study and in particular to Martin Schaich and Oliver Reuss for their support during the project.

We would also like to show our gratitude to the Bavarian State Archaeological Collection and Archäologische Staatssammlung München, responsible for the findings in Ilmendorf, and in particular to Constanze Schaaff for the continuous support during the acquisition and the post-processing phase.

Finally, we would like to thank our academic supervisors for their comments and insights: Fabio Remondino (FBK), Andreas Georgopoulos (NTUA), Livio de Luca (CNRS MAP), Dieter Fritsch (University of Stuttgart), Martin Doerr (ICS-FORTH), Martin Schaich (Arctron3D GmbH), Pedro Santos (Fraunhofer IGD), Alan Chalmers (University of Warwick), Michael Klein (7reasons Medien GmbH), Roko Zarnic (University of Ljubljana).

\section{REFERENCES}

Banterle, F., Artusi, A., Debattista, K., Chalmers, A., 2011. Advanced High Dynamic Range Imaging. A K Peters, Ltd., Natick, MA, USA.

Claßen, E., Gebhard, R., Wiedmann, S., 2010. Fürstin Priesterin - Händlerin: Wer bin ich in der Hallstattzeit? Archäologie in Deutschland 26, pp. 24-27.

Claßen, E., Wiedmann, S., Herzig, F., 2009. Mit reichem Schmuck ins Jenseits - Ein hallstattzeitliches Grab in Ilmendorf. Das Archäologische Jahr in Bayern, pp. 62-65.

Chinosi, M., Trombetta, A., 2012. BPMN: An introduction to the standard. Computer Standards \& Interfaces 34, pp. 124134.

D'Andrea, A., Fernie, K., 2013. CARARE 2.0: A metadata schema for 3D cultural objects, Proceedings. IEEE Conference "Digital Heritage 2013", pp. 137-143.

Fernie, K., Gavrilis, D., Angelis, S., 2013. The CARARE metadata schema, v. 2.0.

Nony, N., De Luca, L., Godet, A., Pierrot-Deseilligny, M., Remondino, F., Van Dongen, A., Vincitore, M., 2012. Protocols and Assisted Tools for Effective Image-Based Modeling of Architectural Elements. Proc. EuroMed 2012 Conference, LNCS 7616, pp. 432-439. Springer, Heidelberg.

Pierrot-Deseilligny, M., De Luca, L., Remondino, F., 2011. Automated image-based procedures for accurate artifacts $3 \mathrm{D}$ modeling and orthoimage generation. Geoinformatics FCE CTU 6, pp. 291-299.

Reinhard, E., Stark, M., Shirley, P., \& Ferwerda, J. (2002, July). Photographic tone reproduction for digital images. In $A C M$ Transactions on Graphics (TOG)(Vol. 21, No. 3, pp. 267-276). ACM.

Remondino, F., El-Hakim, S., 2006. Image-based 3D Modelling: A Review. The Photogrammetric Record 21, pp. 269-291.

Remondino, F., Menna, F., Koutsoudis, A., Chamzas, C., ElHakim, S., 2013. Design and implement a reality-based 3D digitisation and modelling project. Proceedings. IEEE Conference "Digital Heritage 2013", Vol. 1, pp. 137-144

Ronzino, P., Hermon, S., Niccolucci, F., 2012. A metadata schema for cultural heritage documentation. In: Cappellini, V. (Ed.), Electronic Imaging the Visual Arts EVA Florence 2012. Firenze University Press, Firenze, pp. 36-41

Rothermel, M., Wenzel, K., Fritsch, D., 2012. SURE: Photogrammetric surface reconstruction from imagery. Presented at the $L C 3 D$ Workshop, Berlin.

White, S. A., 2004. Introduction to BPMN. Business Process Trends. Available from < http://bptrends.com/> 